\title{
Long-term Exercise After Pulmonary Rehabilitation (LEAP): a pilot randomised controlled trial of Tai Chi in COPD
}

\author{
Marilyn L. Moy $\mathbb{C}^{1,2}$, Peter M. Wayne ${ }^{3,4}$, Daniel Litrownik $^{5}$, Douglas Beach ${ }^{6}$, Elizabeth S. Klings ${ }^{7}$, \\ Roger B. Davis ${ }^{5}$, Adlin Pinheiro ${ }^{5}$ and Gloria Y. Yeh ${ }^{3,5}$
}

${ }^{1}$ Pulmonary, Sleep, and Critical Care Medicine Section, Dept of Medicine, Veterans Administration Boston Healthcare System, Boston, MA, USA. ${ }^{2}$ Harvard Medical School, Boston, MA, USA. ${ }^{3}$ Osher Center for Integrative Medicine, Brigham and Women's Hospital and Harvard Medical School, Boston, MA, USA. ${ }^{4}$ Division of Preventive Medicine, Brigham and Women's Hospital, Boston, MA, USA. ${ }^{5}$ Division of General Medicine, Dept of Medicine, Beth Israel Deaconess Medical Center, Brookline, MA, USA. ${ }^{6}$ Division of Pulmonary, Sleep and Critical Care Medicine, Dept of Medicine, Beth Israel Deaconess Medical Center, Boston, MA, USA. ${ }^{7}$ The Pulmonary Center, Boston University School of Medicine, Boston, MA, USA.

Corresponding author: Marilyn L. Moy (E-mail: marilyn.moy@va.gov)

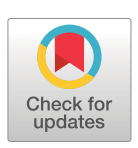

Copyright @The authors 2021

This version is distributed under the terms of the Creative Commons Attribution Non-Commercial Licence 4.0. For commercial reproduction rights and permissions contact permissions@ersnet.org

This article has supplementary material available from openres.ersjournals.com

Received: 12 Jan 2021 Accepted: 24 April 2021

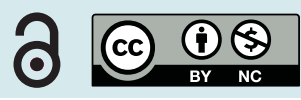

Shareable abstract (@ERSpublications)

Tai Chi, a mind-body modality, may be a feasible option to maintain the benefits gained in exercise capacity and health-related quality of life after completion of outpatient conventional pulmonary rehabilitation https://bit.ly/32YI7Zp

Cite this article as: Moy ML, Wayne PM, Litrownik D, et al. Long-term Exercise After Pulmonary Rehabilitation (LEAP): a pilot randomised controlled trial of Tai Chi in COPD. ERJ Open Res 2021; 7 : 00025-2021 [DOI: 10.1183/23120541.00025-2021].

Abstract

Mind-body modalities are promising strategies to maintain the benefits gained after completion of conventional pulmonary rehabilitation in persons with COPD.

In this pilot randomised controlled study we examined Tai Chi in persons with COPD after completing pulmonary rehabilitation. Participants were randomised 2:2:1 to Tai Chi (TC), usual care (UC) or group walking (GW) for 24 weeks. We assessed feasibility; primary outcome was exercise capacity measured by 6-min walk test (6MWT) distance at 24 weeks. Secondary outcomes included health-related quality of life measured by Chronic Respiratory Questionnaire (CRQ), dyspnoea, mood, stress, social support, selfefficacy, physical activity and exercise engagement. Effect size estimates and estimates from generalised estimating equations were calculated.

Ninety-one persons (36 TC, 37 UC, 18 GW) were enrolled, with mean age $69 \pm 6$ years, 59\% male, and forced expiratory volume in $1 \mathrm{~s} \%$ predicted $\left(\mathrm{FEV}_{1} \%\right.$ pred) $48 \pm 19 \%$. There was no difference in adherence and adverse events between groups. There was a small between-group effect size (ES=0.25) in change in 6MWT distance favouring TC compared to UC; 24-week comparison was nonsignificant $(p=0.10)$. There were no differences in secondary outcomes. In exploratory analyses, there was a greater percentage of participants in TC who improved 6MWT distance at 24 weeks, compared to UC, $64 \%$ versus $39 \%, p=0.05$. There were higher percentages of participants in TC who improved CRQ Fatigue (59\% versus $31 \%, \mathrm{p}=0.02$ ) and CRQ Mastery ( $47 \%$ versus $20 \%$, $\mathrm{p}=0.01)$ domain scores, compared to UC. For $\mathrm{GW}$, there were no differences compared with TC.

Tai Chi may be a feasible option to maintain the benefits gained after completing conventional pulmonary rehabilitation.

\section{Introduction}

The Global Initiative for Chronic Obstructive Lung Disease (GOLD) guidelines recommend regular physical activity for all patients with stable COPD [1]. Conventional pulmonary rehabilitation programmes clearly improve exercise capacity, decrease breathlessness and improve health-related quality of life (HRQL) in COPD [2]. Despite the short-term improvements, the benefits diminish to pre-intervention levels within 6-12 months of programme completion without a maintenance exercise regimen [3-6].

A key goal of conventional pulmonary rehabilitation is to effect behaviour change so patients will engage in long-term exercise [2]. Maintenance strategies to continue exercise after pulmonary rehabilitation programme 
completion can decrease risk for acute exacerbations (AEs) and AE-related hospitalisations [7, 8]. However, there is currently no commonly accepted strategy to maintain exercise in the post-rehabilitation period. Studies of exercise maintenance programmes, with various combinations of supervised exercise classes, unsupervised home exercise, support groups and/or telephone contacts with healthcare professionals, have shown mixed results in persons with COPD [9-13]. Non-adherence to maintenance exercise has been attributed to the occurrence of AEs, depression, lack of exercise opportunities and low self-efficacy [14-16]. Novel exercise options that integrate both physical and psychosocial components are needed to maintain the benefits of pulmonary rehabilitation.

With origins in traditional Chinese martial arts, Tai Chi (TC) is a gentle, conditioning exercise that coordinates physical movements with meditative attention and breathing $[17,18]$. TC integrates three important components of pulmonary rehabilitation: aerobic exercise, dyspnoea management and stress/ anxiety management. TC is relatively low-cost, uses no special equipment and requires minimal space [19], all characteristics that foster long-term adherence. We have developed a protocol of TC movements specifically for persons with COPD [20].

In this study, Long-term Exercise After Pulmonary Rehabilitation (LEAP), we examine the role of TC to maintain the expected benefits gained after completion of conventional pulmonary rehabilitation in persons with COPD. In addition to measures of feasibility, the primary outcome was 6-min walk test (6MWT) distance, compared to usual care at 24 weeks. We further explore and describe changes with TC compared to group walking.

\section{Methods}

\section{Study design}

Details on study design and intervention groups have been previously described [21]. In this pilot randomised controlled study, participants were randomised 2:2:1 to either Tai Chi (TC), usual care (UC) or group walking (GW) for 24 weeks. The cohort was characterised with demographic information and clinical history including cigarette smoking (pack-years), supplemental oxygen use, the Charlson Comorbidity Index and median days since completion of pulmonary rehabilitation at the time of study enrolment [21]. All outcomes were assessed at entry to the research study (i.e. baseline testing which occurred after the completion of the pulmonary rehabilitation programme), at 12 weeks and 24 weeks. Participants were followed post-intervention for an additional 6 months by telephone to assess for the occurrence of AEs at 9 months and secondary outcomes at 1 year.

\section{Study population and recruitment}

Participants with COPD were enrolled from the outpatient pulmonary rehabilitation programmes at five institutions in Massachusetts, United States: Beth Israel Deaconess Medical Center, VA Boston Healthcare System, Brigham and Women's Hospital, Boston Medical Center, and South Shore Hospital. All pulmonary rehabilitation programmes contained the core components of aerobic exercise (treadmill, stationary bicycle, arm ergometer), strength training (resistance bands, free weights) and education delivered by a multidisciplinary staff. To be pragmatic and mirror real-world community pulmonary rehabilitation programmes, we did not require sites to convert to a standardised frequency or duration of classes.

Recruitment occurred between October 2013 and July 2017; participants were enrolled in cohorts. See supplementary material for eligibility criteria. Study staff approached patients during pulmonary rehabilitation classes or at their pulmonary rehabilitation discharge visit. Usual procedures of each pulmonary rehabilitation programme were maintained to educate participants on transitioning to post-rehabilitation exercise. Participants were asked not to start TC on their own or another course of pulmonary rehabilitation programme while enrolled in the study. Ethics approval was obtained at each institution, and written informed consent obtained from each participant.

\section{Randomisation and allocation concealment}

Group assignments were generated by a permuted blocks method with randomly varying block size to ensure balanced but unpredictable assignments. Assignments were sealed in numbered, opaque envelopes. All outcomes testing was conducted by study staff blinded to treatment assignment.

\section{Intervention groups}

Participants in all three groups received written instructions on exercising at home and disease self-management. Both TC and GW classes were conducted at a local university fitness facility. TC instructors led the TC classes and trained study staff led the GW classes. 
Tai Chi

TC included 36 classes of 1-h duration with 2 classes per week for 12 weeks, then weekly for 12 additional weeks. The TC intervention was designed specifically for an older, physically limited population [20, 22-24]. A guided audio CD of TC exercises and an instructional TC DVD facilitated home practice. Participants were encouraged to practice TC outside of class at least three times a week for 30 min each time.

\section{Usual care}

As part of usual procedures of each pulmonary rehabilitation programme, upon completion of the supervised, facility-based programme, all participants met with pulmonary rehabilitation staff to formulate an unsupervised exercise plan to follow at home [2]. Participants were also allowed to continue in the maintenance programmes of their usual pulmonary rehabilitation programme.

\section{Group walking}

The supervised GW classes were identical to the TC classes in terms of duration, number and frequency, and type of physical activity (low-moderate aerobic exercise with gentle stretching). Participants walked at their own pace around an indoor gym, targeting the intensity of exercise to reach $\sim 60 \%$ of their maximum heart rate and to keep breathlessness within the 3-5 range on the Borg scale [25]. Like TC, participants were instructed to walk and perform stretches at least three times per week for 30 min outside of group classes. GW received written handout instructions for stretches that they performed in GW classes.

\section{Outcome measures}

Exercise capacity was assessed with the 6MWT which was conducted at all sites using scripted instructions according to American Thoracic Society (ATS) guidelines, except that a practice walk was not administered [26]. The minimal clinically important change in 6MWT distance is $30-54 \mathrm{~m}$ in stable COPD $[27,28]$. See supplementary material for details about secondary outcomes and other testing. Outcomes were assessed by the Chronic Respiratory Disease Questionnaire (CRQ) and its four domains of dyspnoea, fatigue, emotional function and mastery; University of California, San Diego Shortness of Breath (UCSD SOB) Questionnaire; Center of Epidemiology Studies-Depression Scale (CES-D); Perceived Stress Scale (PSS); Multidimensional Scale of Perceived Social Support (MSPSS); COPD Self-Efficacy Scale (CSES); Resnick Exercise Self-Efficacy; and Community Health Activities Model Programme for Seniors (CHAMPS) Physical Activity Questionnaire for Older Adults. The Omron HJ-720ITC pedometer objectively measured physical activity as daily step counts. Exercise logs captured exercise engagement. A composite measure of exercise engagement was calculated using total minutes of class time, home practice time and other physical activity on the exercise logs.

\section{Other data collection}

Study adherence

Participants with attendance of $>70 \%$ of TC or GW classes were defined a priori as being adherent. We also compared self-reported home practice time of TC or walking to the expected time as provided in study instructions, and the number of study visits at each timepoint completed by each participant.

\section{Adverse events}

At each visit every 3 months, participants were queried about new or worsening symptoms and medical conditions, change in medications, or urgent care visits, emergency room visits or hospitalisations. Participants enrolled in TC and GW also completed logs asking about adverse events, which were collected at every class attended.

\section{Statistical analysis}

While an initial primary aim was to assess feasibility, the study was also powered on the 6MWT distance and between-group comparisons were proposed. We recognise that sample size estimates, largely based on one small study [29, 30], may be misleading. Thus, we have chosen to primarily present effect size (ES) estimates and calculation of Cohen's d. Hedges and Olkin's formula calculated 95\% confidence intervals for Cohen's d [31]. The magnitude of effect sizes of 0.2, 0.5 and 0.8 represent small, medium and large effect sizes, respectively [32]. We primarily focused on comparisons at 24 weeks between TC and UC.

Generalised estimating equations methods accounting for repeated measures also provided estimates of the difference in mean changes in outcomes between groups. Models were adjusted for baseline values that were imbalanced despite randomisation: sex, time since completion of pulmonary rehabilitation, supplemental oxygen use and Charlson Comorbidity Index. 
Improvement in all outcomes was defined by any change that was better than the baseline value. Changes in the primary outcome of 6MWT distance at 24 weeks were analysed using a non-parametric WilcoxonMann-Whitney test. In addition, as further exploratory analyses, we examined outcomes as dichotomised variables: those who had any improvement versus those with no change or worsened. Chi-squared tests compared the dichotomised variables between groups. Analyses were performed using SAS, version 9.4 (SAS Institute Inc., Cary, NC).

\section{Results}

\section{Study population and baseline characteristics}

Ninety-one persons with COPD who had completed pulmonary rehabilitation were enrolled (N=36 TC, $\mathrm{N}=37 \mathrm{UC}, \mathrm{N}=18 \mathrm{GW}$ ) (figure 1). Mean age was $69 \pm 6$ years, $59 \%$ were male, mean forced expiratory volume in $1 \mathrm{~s} \%$ predicted $\left(\mathrm{FEV}_{1} \%\right.$ pred) was $48 \pm 19 \%$, and $58 \%$ were GOLD stage 3-4 [1]. Mean pack-years was $51 \pm 29$, and $37 \%$ were on supplemental oxygen. In terms of self-reported comorbidities, $25 \%$ had cardiovascular disease, 31\% cancer and 53\% chronic musculoskeletal issues or back pain.

There were slight imbalances between groups at baseline with respect to sex, Charlson Comorbidity Index, supplemental oxygen use and time since completion of pulmonary rehabilitation. There were more males randomised to UC, compared to TC and GW (73\% versus 50\% versus $47 \%$, respectively). There was also a trend towards more participants regularly using supplemental oxygen in TC, compared to GW and UC (47\% versus $39 \%$ versus $27 \%$ ) (table 1 ).

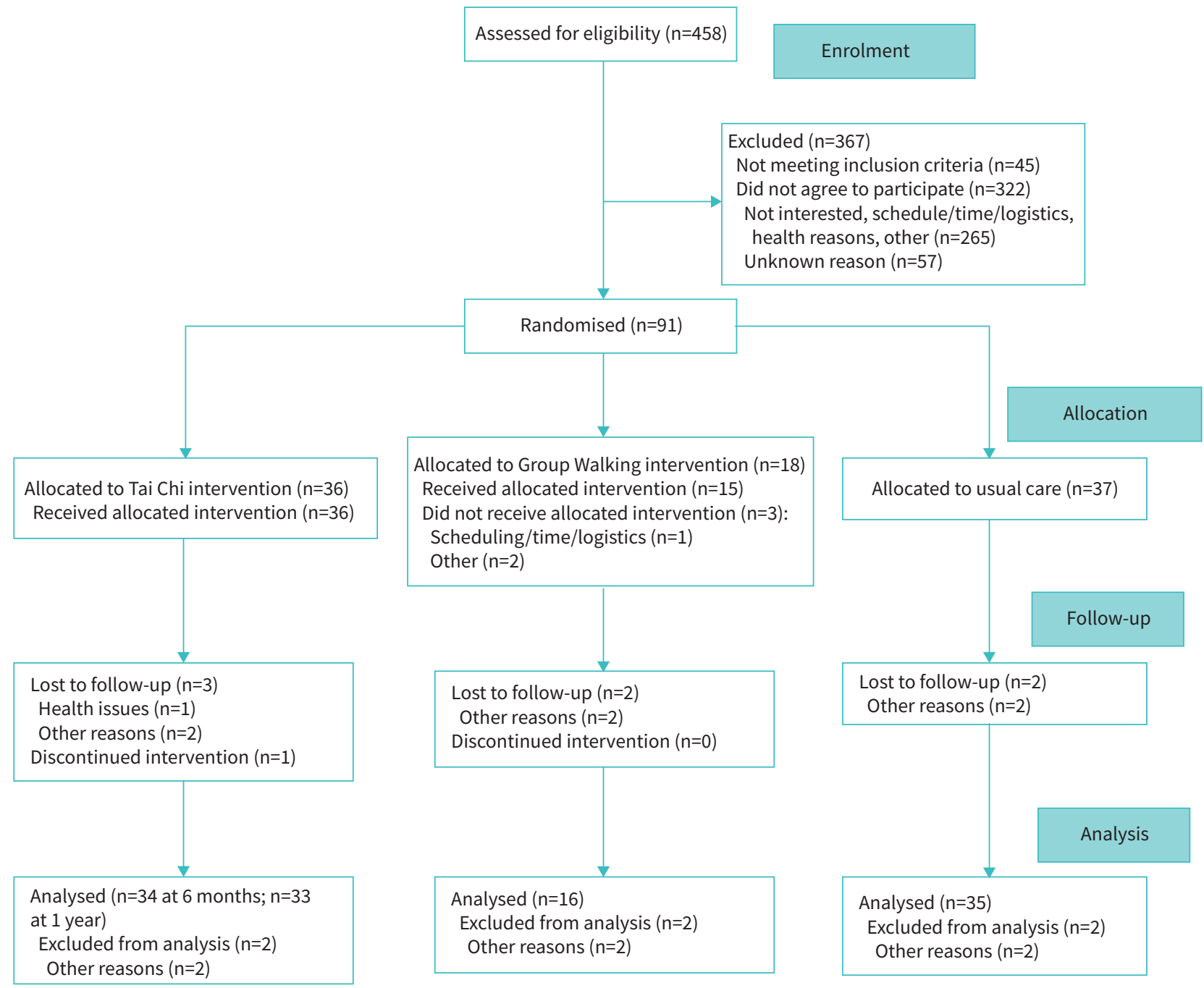

FIGURE 1 Consolidated Standards of Reporting Trials flow chart. 


\begin{tabular}{|c|c|c|c|}
\hline Characteristic & Tai Chi & Group walking & Usual care \\
\hline Subjects $\mathrm{n}$ & 36 & 18 & 37 \\
\hline Age years & $69.6 \pm 7.5$ & $66.9 \pm 6.7$ & $70.5 \pm 9.2$ \\
\hline Male sex & $17(47)$ & $9(50)$ & $27(73)$ \\
\hline \multicolumn{4}{|l|}{ Race } \\
\hline White & $28(78)$ & $12(67)$ & $27(73)$ \\
\hline Black & $6(17)$ & $3(17)$ & $8(22)$ \\
\hline Other/unknown & $2(6)$ & $3(17)$ & $2(6)$ \\
\hline Annual income less than USD 35000 & $16(44)$ & $7(39)$ & $15(41)$ \\
\hline Married or living with partner & $13(36)$ & $8(44)$ & $18(49)$ \\
\hline Unemployed, retired or disabled & $32(89)$ & $16(89)$ & $35(95)$ \\
\hline $\mathrm{FEV}_{1} \%$ predicted & $48.1 \pm 17.9$ & $50.8 \pm 24.6$ & $45.7 \pm 17.7$ \\
\hline \multicolumn{4}{|l|}{ GOLD stage } \\
\hline Stage $1-2$ & $16(44)$ & $10(56)$ & $13(35)$ \\
\hline Stage $3-4$ & $20(56)$ & $8(44)$ & $24(65)$ \\
\hline \multicolumn{4}{|l|}{ BODE index } \\
\hline $0-2$ & $13(36)$ & $7(39)$ & $14(38)$ \\
\hline $3-4$ & $16(44)$ & $4(22)$ & $14(38)$ \\
\hline 5 or greater & $7(19)$ & $7(39)$ & $9(24)$ \\
\hline Regular oxygen use & $17(47)$ & $7(39)$ & $10(27)$ \\
\hline Smoking pack-years & $52 \pm 33.7$ & $44.9 \pm 24.8$ & $54 \pm 26.5$ \\
\hline Days since completing pulmonary rehabilitation, median (IQR) & $35(99)$ & $62(120)$ & $72(126)$ \\
\hline Charlson Comorbidity Index & $7.3 \pm 2.5$ & $6.7 \pm 2.8$ & $6.4 \pm 2.4$ \\
\hline \multicolumn{4}{|l|}{ Comorbidities } \\
\hline Cardiovascular disease & $8(22)$ & $4(22)$ & $10(27)$ \\
\hline Heart failure & $6(17)$ & $2(11)$ & $7(19)$ \\
\hline Cancer & $13(36)$ & $6(33)$ & $9(24)$ \\
\hline Hypertension & $24(67)$ & $10(56)$ & $25(68)$ \\
\hline Limitation of limb" & $2(6)$ & $3(17)$ & $3(8)$ \\
\hline Osteoarthritis, sciatica or chronic back pain & $19(53)$ & $11(61)$ & $18(49)$ \\
\hline Peripheral vascular disease & $4(11)$ & $4(22)$ & $4(11)$ \\
\hline Stroke or cerebrovascular disease & $6(17)$ & 0 & $4(11)$ \\
\hline 6-min walk test distance $\mathrm{m}$ & $324.7 \pm 123.4$ & $365.3 \pm 105.6$ & $369.7 \pm 104.7$ \\
\hline CES-D & $12.2 \pm 9.2$ & $11.1 \pm 8.1$ & $11.3 \pm 9.3$ \\
\hline \multicolumn{4}{|c|}{$\begin{array}{l}\text { Data are presented as } \mathrm{n}(\%) \text { or mean } \pm \mathrm{SD} \text {, unless otherwise stated. } \mathrm{FEV}_{1} \text { : forced expiratory volume in } 1 \mathrm{~s} \text {; GOLD: } \\
\text { Global Initiative for Chronic Obstructive Lung Disease; BODE: body mass index, airflow obstruction, dyspnoea, } \\
\text { exercise capacity; IQR: interquartile range; CES-D: Center of Epidemiology Studies-Depression Scale. \#: paralysis } \\
\text { or weakness. }\end{array}$} \\
\hline
\end{tabular}

\section{Study adherence}

Average class attendance was $81 \%$ in TC and $64 \%$ in GW. According to our definition of adherence, $83 \%$ in $\mathrm{TC}$ and $50 \%$ in $\mathrm{GW}$ attended $\geqslant 70 \%$ of classes. In the first 12 weeks, participants in TC reported $80 \pm 55 \mathrm{~min} \cdot$ week $^{-1}$ of home practice, and those in GW $73 \pm 48 \mathrm{~min} \cdot \mathrm{week}^{-1}$. In the second 12 weeks, participants reported $46 \pm 48$ and $41 \pm 39 \mathrm{~min} \cdot$ week $^{-1}$ of home practice in TC and GW, respectively.

At 12 weeks, 86 participants and at 24 weeks, 85 participants completed the follow-up visits. In total, 84 subjects (93\%) completed outcome testing at 1 year; 7\% were lost to follow-up (3 in TC, 2 in UC and 2 in GW). There were no significant differences in baseline characteristics between those lost to follow-up versus those who completed the study.

Safety

Thirty-one adverse events occurred during the 24-week intervention period (19 in TC, 6 in GW, 6 in UC). In the subsequent 24-week follow-up period, there were 27 adverse events (8 in TC, 7 in GW, 12 in UC). Over the 1-year study, there were 32 respiratory-related events (15 in TC, 7 in GW, 10 in UC). One participant in GW experienced musculoskeletal knee pain which was related to the study.

COPD acute exacerbations during the 24-week intervention period included 19 in TC, 8 in GW and 20 in UC. The percentage of subjects with at least one AE who required a course of antibiotics or corticosteroids 
was $9 \%$ in TC, $19 \%$ in GW and $20 \%$ in UC. There were no significant differences between groups with respect to total number of AEs or number of participants with an AE at 3, 6, 9 or 12 months.

\section{Exercise capacity}

There was a small between-group effect size $(\mathrm{ES}=0.25)$ describing change in 6MWT distance favouring an increase in TC compared to UC at 24 weeks, although a Wilcoxon-Mann-Whitney test was not significant $(\mathrm{p}=0.10)$ (table 2). There was a significantly greater percentage of participants in TC who improved $6 \mathrm{MWT}$ distance at 24 weeks, compared to UC, $64 \%$ versus $39 \%, \mathrm{p}=0.05$. Within TC, there was a $43-\mathrm{m}$ increase in 6MWT distance at 12 weeks, compared to baseline $(\mathrm{p}=0.07)$, which decreased to a $22-\mathrm{m}$ change at 24 weeks, $\mathrm{p}=0.17$. In contrast, $\mathrm{GW}$ and UC had nonsignificant decreases from baseline to 24 weeks ( $27 \mathrm{~m}$ in $\mathrm{GW}, \mathrm{p}=0.50$, and $17 \mathrm{~m}$ in $\mathrm{UC}, \mathrm{p}=0.17$ ).

\section{HRQL, dyspnoea, mood, stress, social support and self-efficacy}

At 24 weeks and 1 year, there were small effect sizes favouring TC over UC in CRQ Total, CRQ Fatigue, CRQ Mastery and COPD Self-Efficacy (ES=0.2-0.46) (table 2). Examining CRQ scores as dichotomised outcomes, there were higher percentages of participants in TC who improved their CRQ Fatigue (59\% versus $31 \%, \mathrm{p}=0.02)$ and CRQ Mastery $(47 \%$ versus $20 \%, \mathrm{p}=0.01)$ domain scores, compared to UC, at 24 weeks.

Within TC, there were improvements in CRQ Total at 12 weeks compared to baseline $(+0.22 \pm 0.70$, $\mathrm{p}=0.04$ ) but no change at 24 weeks or 1 year. At 24 weeks, there were also within-group improvements in TC in COPD self-efficacy $(+0.22 \pm 0.64, \mathrm{p}=0.05)$, social support $(+0.22 \pm 1.26, \mathrm{p}=0.04)$ and perceived stress $(-2.47 \pm 4.76, \mathrm{p}=0.01)$. No changes in TC were seen in UCSD SOB score or CES-D score at 12 and 24 weeks, and at 1 year. In GW or UC, there were no significant changes in secondary outcomes at any follow-up time compared to baseline.

Examining social support as a dichotomised outcome, there was a significantly higher percentage of participants in TC who improved their MSPSS score (62\% versus 25\%, p=0.02), compared to GW, at 24 weeks.

\section{Physical activity and exercise engagement}

At 24 weeks, the mean daily step count was $2431 \pm 1357$ in TC, $2839 \pm 2376$ in GW and $3294 \pm 2461$ in UC (table 3). At 1 year, daily step count was $2264 \pm 1255$ in TC, $2983 \pm 3549$ in GW and $3402 \pm 2884$ in UC. In the subgroup of participants who wore the Omron pedometer at both follow-up timepoints, there was no difference in physical activity directly measured as daily step counts between the three groups.

Although TC did not increase daily step counts during the 24 weeks, participants appeared to be doing other physical activities and engaging in TC as demonstrated by the class attendance and exercise engagement data (table 3). There was reasonable home practice in TC; they were encouraged to engage in TC three times/week for $30 \mathrm{~min}$ each session, and the data showed an average of $80 \mathrm{~min} /$ week and 3.9 times/week. UC did more walking, strength training and cardiovascular exercise (i.e. what they had learned in pulmonary rehabilitation) while TC performed more everyday activities (i.e. housework) and sports. Overall, there were no differences between TC and UC with respect to total self-reported minutes of physical activity at 12 and 24 weeks, and at 12 months (table 3). Composite exercise engagement, including intervention, home practice and other self-reported physical activity was higher in the first 12 weeks in TC, but similar to the other two groups in the follow-up periods.

\section{Discussion}

The Long-term Exercise After Pulmonary Rehabilitation (LEAP) study examined TC to maintain benefits after completion of outpatient conventional pulmonary rehabilitation in persons with COPD. There was a small between-group effect size in change in 6MWT distance favouring TC compared to UC, although the comparison was not statistically significant. In exploratory analyses, there was a significantly greater percentage of persons in TC who had an improvement in exercise capacity, compared to UC. There were also significantly higher percentages of participants in TC who improved HRQL domains, such as fatigue and mastery, compared to UC at 24 weeks. TC was safe, without increased numbers of adverse events, compared to UC. In terms of adherence, class attendance was better in TC compared to GW, but home practice was similar.

Maintenance exercise regimens have typically focused on supervised exercise training with walking drills or cycle ergometers for leg training, and free weights for upper extremity training [3-9, 11-13]. Unlike these previous maintenance regimens that have improved exercise capacity but not HRQL, TC also 
TABLE 2 Mean changes in 6-min walk test (6MWT) distance and secondary outcomes

\begin{tabular}{|c|c|c|c|c|c|c|c|c|c|c|}
\hline \multirow[t]{2}{*}{ Outcome measure } & \multicolumn{2}{|c|}{ Baseline values } & \multicolumn{3}{|c|}{ Change from baseline to 24 weeks } & \multirow{2}{*}{$\begin{array}{c}\text { Adjusted mean difference } \\
\text { at week } 24\end{array}$} & \multicolumn{3}{|c|}{ Change from baseline to 1 year } & \multirow{2}{*}{$\begin{array}{c}\text { Adjusted mean difference } \\
\text { at } 1 \text { year }\end{array}$} \\
\hline & Tai Chi & UC & Tai Chi & UC & Effect size & & Tai Chi & UC & Effect size & \\
\hline 6MWT distance $\mathrm{m}$ & $324.7 \pm 123.4$ & $369.7 \pm 104.7$ & $6.8 \pm 53.3$ & $-5.1 \pm 41.2$ & $0.25(-0.24-0.73)$ & $3.2(-21.1-27.4)$ & & & & \\
\hline CRQ dyspnoea & $4.97 \pm 1.38$ & $5.67 \pm 1.01$ & $0.06 \pm 1.1$ & $-0.12 \pm 1.06$ & $0.17(-0.31-0.64)$ & $-0.17(-0.66-0.32)$ & $-0.16 \pm 1.13$ & $-0.31 \pm 1.12$ & $0.14(-0.34-0.61)$ & $-0.20(-0.75-0.35)$ \\
\hline CRQ fatigue & $4.30 \pm 1.06$ & $4.91 \pm 0.96$ & $0.21 \pm 1.03$ & $-0.16 \pm 0.83$ & $0.40(-0.08-0.87)$ & $0.17(-0.28-0.62)$ & $0.08 \pm 0.96$ & $-0.23 \pm 0.88$ & $0.33(-0.15-0.81)$ & $0.10(-0.31-0.5)$ \\
\hline CRQ emotion & $5.24 \pm 1.05$ & $5.51 \pm 1.14$ & $-0.06 \pm 0.69$ & $0.05 \pm 0.63$ & $-0.18(-0.65-0.3)$ & $-0.24(-0.57-0.1)$ & $0.01 \pm 0.91$ & $-0.25 \pm 0.78$ & $0.3(-0.17-0.78)$ & $0.17(-0.21-0.56)$ \\
\hline CRQ mastery & $5.36 \pm 1.44$ & $5.66 \pm 1.22$ & $0.14 \pm 1.05$ & $-0.14 \pm 0.77$ & $0.30(-0.18-0.77)$ & $-0.11(-0.51-0.3)$ & $0.06 \pm 1.29$ & $-0.24 \pm 1.04$ & $0.26(-0.22-0.74)$ & $0.06(-0.39-0.51)$ \\
\hline CRQ total & $5.01 \pm 0.98$ & $5.46 \pm 0.93$ & $0.06 \pm 0.73$ & $-0.07 \pm 0.62$ & $0.20(-0.27-0.67)$ & $-0.08(-0.42-0.27)$ & $-0.01 \pm 0.81$ & $-0.26 \pm 0.72$ & $0.33(-0.15-0.81)$ & $0.08(-0.29-0.45)$ \\
\hline $\begin{array}{l}\text { UCSD shortness of } \\
\text { breath }\end{array}$ & $39.54 \pm 19.42$ & $29.58 \pm 14.7$ & $-0.22 \pm 11.24$ & $-0.09 \pm 11.78$ & $-0.01(-0.48-0.46)$ & $2.05(-4.26-8.37)$ & $2.53 \pm 12.65$ & $0.7 \pm 14.09$ & $0.14(-0.34-0.61)$ & $3.79(-3.27-10.85)$ \\
\hline COPD self-efficacy & $3.38 \pm 0.83$ & $3.64 \pm 0.78$ & $0.22 \pm 0.64$ & $0.02 \pm 0.57$ & $0.34(-0.14-0.81)$ & $0.14(-0.11-0.39)$ & $0.29 \pm 0.6$ & $0.01 \pm 0.65$ & $0.46(-0.02-0.94)$ & $0.21(-0.06-0.49)$ \\
\hline CES-D score & $12.18 \pm 9.22$ & $11.31 \pm 9.28$ & $-1.21 \pm 4.48$ & $-1.54 \pm 4.91$ & $0.07(-0.4-0.54)$ & $0.83(-1.23-2.89)$ & $-0.82 \pm 5.72$ & $-0.37 \pm 6.93$ & $-0.07(-0.55-0.41)$ & $0.13(-2.51-2.78)$ \\
\hline MSPSS social support & $5.64 \pm 1.33$ & $5.72 \pm 1.14$ & $0.22 \pm 1.26$ & $0.17 \pm 1.04$ & $0.04(-0.43-0.51)$ & $-0.06(-0.44-0.31)$ & $0.33 \pm 0.84$ & $0.22 \pm 1.03$ & $0.12(-0.35-0.6)$ & $0(-0.29-0.3)$ \\
\hline PSS perceived stress & $11.73 \pm 6.32$ & $11.1 \pm 5.75$ & $-2.47 \pm 4.76$ & $-0.27 \pm 4.56$ & $-0.47(-0.95-0.01)$ & $-1.40(-3.51-0.71)$ & $-0.10 \pm 5.41$ & $0.83 \pm 4.97$ & $-0.18(-0.66-0.3)$ & $-0.37(-2.66-1.92)$ \\
\hline $\begin{array}{l}\text { Resnick exercise } \\
\text { self-efficacy }\end{array}$ & $57.59 \pm 19.28$ & $66.23 \pm 23.56$ & $-10.69 \pm 21.52$ & $-2.97 \pm 18.62$ & $-0.38(-0.86-0.09)$ & $-11.64(-21.44--1.83)$ & $-12.2 \pm 21.81$ & $-9.34 \pm 23.89$ & $-0.13(-0.6-0.35)$ & $-7.05(-17.01-2.91)$ \\
\hline $\begin{array}{l}\text { Calorie expenditure - } \\
\text { moderate intensity }\end{array}$ & $1561 \pm 3390$ & $1317 \pm 1969$ & $65 \pm 2276$ & $614 \pm 2636$ & $-0.22(-0.79-0.35)$ & $-242(-1789-1305)$ & $634 \pm 3940$ & $-558 \pm 1321$ & $0.41(-0.18-0.99)$ & $1546(-20-3111)$ \\
\hline Daily step count & $2623 \pm 1597$ & $3205 \pm 2265$ & $-404 \pm 749$ & $146 \pm 1667$ & $-0.43(-1.01-0.16)$ & $-560(-1190-70)$ & $-401 \pm 788$ & $-206 \pm 1819$ & $-0.14(-0.72-0.44)$ & $-225(-863-414)$ \\
\hline
\end{tabular}

Data are presented as mean \pm SD change or standardised difference $(95 \% \mathrm{CI})$. Each row represents one model that incorporates all time points. Models adjust for time since completion of pulmonary rehabilitation, supplemental oxygen use, sex and Charlson Comorbidity Index. For measures where higher score means better status, positive values reflect improvement, while negative values indicate deterioration. For measures where higher score reflects worse status, positive values reflect deterioration while negative values indicate improvement. UC: usual care; CRQ: Chronic Respiratory Questionnaire; UCSD: University of California, San Diego; CES-D: Center of Epidemiology Studies-Depression Scale; MSPSS: Multidimensional Scale of Perceived Social Support; PSS: Perceived Stress Scale. 
TABLE 3 Adherence, physical activity and exercise engagement

\begin{tabular}{|c|c|c|c|c|c|c|c|c|c|}
\hline & \multicolumn{3}{|c|}{ Baseline to 12 weeks } & \multicolumn{3}{|c|}{$12-24$ weeks } & \multicolumn{3}{|c|}{24 weeks-1 year } \\
\hline & Tai Chi & Group walking & Usual care & Tai Chi & Group walking & Usual care & Tai Chi & Group walking & Usual care \\
\hline \multicolumn{10}{|l|}{ Adherence } \\
\hline Class time, $\mathrm{min} \cdot$ week $^{-1}$ & $105.3 \pm 33.2$ & $82.5 \pm 43.4$ & & $40.6 \pm 16.3$ & $33.1 \pm 17.2$ & & & & \\
\hline Home practice time, min.week ${ }^{-1}$ & $80.2 \pm 55.2$ & $73.3 \pm 48.3$ & & $45.7 \pm 48.5$ & $40.5 \pm 39.8$ & & & & \\
\hline \multicolumn{10}{|l|}{ Physical activity logs, min·week ${ }^{-1}$} \\
\hline Total & $414.9 \pm 365.6$ & $249.6 \pm 203.0$ & $402.9 \pm 295.5$ & $368.3 \pm 386.5$ & $240.6 \pm 200.3$ & $382.2 \pm 372.9$ & $351.8 \pm 418.0$ & $319.8 \pm 312.2$ & $376.0 \pm 329.2$ \\
\hline Walking & $125.3 \pm 141.6$ & $126.2 \pm 139.5$ & $173.5 \pm 191.9$ & $115.3 \pm 159.6$ & $117.0 \pm 126.6$ & $183.7 \pm 310.8$ & $107.0 \pm 131.4$ & $136.0 \pm 180.4$ & $160.1 \pm 208.5$ \\
\hline Stretching exercises & $17.6 \pm 22.4$ & $21.4 \pm 22.9$ & $27.9 \pm 38.9$ & $15.2 \pm 24.1$ & $16.2 \pm 16.7$ & $27.2 \pm 35.3$ & $11.9 \pm 15.7$ & $26.5 \pm 27.5$ & $29.9 \pm 41.0$ \\
\hline Cardiovascular exercise $^{\#}$ & $9.3 \pm 19.0$ & $2.7 \pm 5.3$ & $37.2 \pm 51.1$ & $4.9 \pm 11.9$ & $1.4 \pm 2.6$ & $35.0 \pm 66.2$ & $10.3 \pm 25.3$ & $7.0 \pm 15.0$ & $26.1 \pm 38.8$ \\
\hline Strength training & $20.9 \pm 37.0$ & $10.0 \pm 15.2$ & $37.5 \pm 60.0$ & $18.0 \pm 28.5$ & $10.0 \pm 18.6$ & $28.1 \pm 40.5$ & $19.8 \pm 33.0$ & $19.8 \pm 26.7$ & $35.0 \pm 57.4$ \\
\hline Playing sports ${ }^{+}$ & $17.0 \pm 62.6$ & $0.2 \pm 0.6$ & $4.3 \pm 23.0$ & $11.0 \pm 32.8$ & 0 & $1.3 \pm 5.0$ & $5.4 \pm 13.3$ & $3.2 \pm 10.3$ & $1.0 \pm 4.7$ \\
\hline Heavy housework ${ }^{\S}$ & $33.2 \pm 69.7$ & $9.2 \pm 20.2$ & $22.4 \pm 38.6$ & $57.8 \pm 172.1$ & $5.6 \pm 9.7$ & $19.4 \pm 30.6$ & $65.9 \pm 231.2$ & $27.3 \pm 29.8$ & $22.6 \pm 57.5$ \\
\hline Light housework $^{f}$ & $132.6 \pm 174.3$ & $69.8 \pm 63.9$ & $88.2 \pm 14.2$ & $95.0 \pm 121.3$ & $70.9 \pm 73.7$ & $78.6 \pm 93.7$ & $96.3 \pm 123.9$ & $92.6 \pm 108.4$ & $90.7 \pm 150.4$ \\
\hline Composite exercise engagement, $\min \cdot$ week $^{-1}$ & $538.7 \pm 366.9$ & $396.3 \pm 220.7$ & $402.9 \pm 295.5$ & $404.7 \pm 397.7$ & $306.3 \pm 193.2$ & $382.1 \pm 373.0$ & $322.2 \pm 419.5$ & $319.8 \pm 312.2$ & $376.0 \pm 329.2$ \\
\hline
\end{tabular}

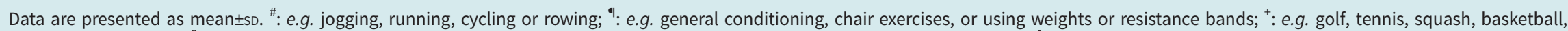

football or swimming; ${ }^{\S}$ : e.g. washing windows, cleaning gutters, home repairs, mowing lawn, raking leaves or shovelling snow; ${ }^{f}$ : e.g. sweeping, vacuuming, cleaning, watering plants, weeding or planting. 
improved HRQL over 24 weeks. These findings are not surprising since TC also incorporates cognitive components, including heightened somatic awareness, focused mental attention, and stress management that can positively impact HRQL.

The significant between-group differences were not observed when change in 6MWT distance or CRQ domains were examined as continuous variables. Nevertheless, given the pilot nature of the study, we noted positive effect sizes for change scores as well as within-group changes in TC, which were not observed in UC or GW, that might inform future studies. Because the elderly commonly choose walking as a convenient and familiar exercise, we explored whether GW, with its added social support, could maintain benefits more effectively than UC. However, these results did not show effect sizes in favour of UC or GW.

Like previous studies, improvements at 24 weeks were no longer evident at 1 year following completion of pulmonary rehabilitation. We also observed a decrease in exercise time in both TC and GW during the second 12 weeks compared to the first 12 weeks, suggesting that long-term sustained behaviour change is also a challenge for mind-body exercise interventions. Diaries, phone calls, pedometers and a web-based app have been previously used to improve adherence [33, 34]. Our data suggest that continued TC classes may be important. As observed in other pulmonary rehabilitation studies, our participants with COPD referred to pulmonary rehabilitation had moderate-severe obstructive lung disease and multiple comorbidities which may interrupt long-term exercise engagement [13].

We note that the study was powered for between-group difference in change in 6MWT distance at 24 weeks compared to baseline (when pulmonary rehabilitation was completed). We hypothesised that the change in 6MWT distance would be greater in the TC group compared to UC. We did not specify which direction of change would occur within each group, but based on the literature about the natural course of exercise capacity after completion of pulmonary rehabilitation, we expected that the UC group would decline, while the TC group would maintain or improve 6MWT distance. Thus, maintenance rather than continued improvement of benefits in the TC group, leading to a significant change compared to UC, can also be viewed as a meaningful outcome.

Given limited exercise options for pulmonary rehabilitation maintenance, these data on TC are very encouraging. It is likely that no one model of exercise maintenance is ideal for all patients with COPD and that personalised maintenance exercise programmes are needed. A broad repertoire of exercise opportunities would optimise maintenance of physical activity and exercise in persons with COPD who have completed pulmonary rehabilitation [16, 33]. Previous studies in persons with COPD who had not already engaged in pulmonary rehabilitation support TC's feasibility, safety and efficacy [35, 36]. The low-impact, adaptable movements and postures make TC particularly attractive for persons with COPD $[37,38]$. In a separate pilot randomised controlled trial (RCT) in patients with moderate-severe COPD, we demonstrated trends toward improvement in HRQL, depression and dyspnoea after 3 months of TC versus an education control group [20]. The current results extend these previous studies by showing that TC may be a feasible option to maintain the benefits gained after completion of supervised pulmonary rehabilitation.

The type, frequency, intensity and duration of exercise needed for optimal maintenance and outcomes are unknown. It appears that longer duration programmes are more beneficial. Supervised maintenance exercise programmes that lasted 9-12 months were associated with risk reduction of pulmonary-related hospitalisations [8]. Improvements in 6MWT distance, CAT score and Modified Medical Research Council (mMRC) dyspnoea score were demonstrated with a 12-month maintenance programme of home visits and telephone contacts [39]. Clearer benefits may have been observed if we had continued our TC intervention to 9-12 months. Based on our findings that UC did more walking, strength training and cardiovascular exercise while TC performed more everyday activities and sports, we acknowledge that more UC participants may have engaged in maintenance pulmonary rehabilitation since they were enrolled in our research study compared to rates typically seen in the clinical setting.

We enrolled a well-characterised cohort of persons with COPD and comprehensively assessed physiological and psychosocial outcomes. Strengths of this study include the RCT design and enrolment of an ethnically diverse Western population. It is possible that only a subset of patients who complete conventional pulmonary rehabilitation would benefit from TC as a maintenance regimen. In a cohort who all improved outcomes after 8 weeks of outpatient pulmonary rehabilitation, mixed results of a maintenance intervention were explained by the fact that not all participants are realistic targets for maintenance exercise given the severity of underlying lung disease and/or comorbidities [13]. It has also been suggested that the degree of improvement in exercise capacity after the initial conventional 
pulmonary rehabilitation programme may affect the ability to achieve long-term benefits with a maintenance regimen [33]. We were unable to stratify by those who improved outcomes after conventional pulmonary rehabilitation, before enrolling in this study. We acknowledge that we have shown statistically significant improvements in outcomes but not necessarily clinically important improvements. Overall, TC may be a feasible option to maintain the benefits gained after completing pulmonary rehabilitation and warrants further study.

Acknowledgements: We thank the study participants and the Tai Chi instructors, Stanwood Chang, Jane Moss and Regina Gibbons.

This study is registered at www.clinicaltrials.gov with identifier number NCT01998724. Data sharing will be granted upon reasonable request directly to the authors.

Conflict of interest: M.L. Moy reports grants from the NIH during the conduct of the study. P.M. Wayne is the founder and sole owner of the Tree of Life Tai Chi Center. His interests were reviewed and are managed by the Brigham and Women's Hospital and Partners HealthCare in accordance with their conflict of interest policy. D. Litrownik reports grants from the NIH during the conduct of the study. D. Beach has nothing to disclose. E.S. Klings has nothing to disclose. R.B. Davis reports grants from the NIH during the conduct of the study. A. Pinheiro has nothing to disclose. G.Y. Yeh reports grants from the NIH during the conduct of the study.

Support statement: This study was supported by an award from the National Center for Complementary and Integrative Health (NCCIH), National Institutes of Health (NIH) (to M.L. Moy and G.Y. Yeh, R01AT006358). Its contents are solely the responsibility of the authors and do not necessarily represent the official views of the $\mathrm{NCClH}$ or the NIH. G.Y. Yeh was supported by NIH K24 AT009465 and P.M. Wayne was supported by NIH K24 AT009282. Funding information for this article has been deposited with the Crossref Funder Registry.

\section{References}

1 Vestbo J, Hurd SS, Agustí AG, et al. Global strategy for the diagnosis, management, and prevention of chronic obstructive pulmonary disease: GOLD executive summary. Am J Respir Crit Care Med 2013; 187: 347-365.

2 Spruit MA, Singh SJ, Garvey C, et al. An official American Thoracic Society/European Respiratory Society statement: key concepts and advances in pulmonary rehabilitation. Am J Respir Crit Care Med 2013; 188: e13-e64.

3 Ries AL, Kaplan RM, Myers R, et al. Maintenance after pulmonary rehabilitation in chronic lung disease: a randomized trial. Am J Respir Crit Care Med 2003; 167: 880-888.

4 Brooks D, Krip B, Mangovski-Alzamora S, et al. The effect of postrehabilitation programmes among individuals with chronic obstructive pulmonary disease. Eur Respir J 2002; 20: 20-29.

5 Güell R, Casan P, Belda J, et al. Long-term effects of outpatient rehabilitation of COPD: a randomized trial. Chest 2000; 117: 976-983.

6 Griffiths TL, Burr ML, Campbell IA, et al. Results at 1 year of outpatient multidisciplinary pulmonary rehabilitation: a randomised controlled trial. Lancet 2000; 355: 362-368.

7 Vasilopoulou M, Papaioannou Al, Kaltsakas G, et al. Home-based maintenance tele-rehabilitation reduces the risk for acute exacerbations of COPD, hospitalisations and emergency department visits. Eur Respir J 2017; 49: 1602129.

8 Jenkins AR, Gowler H, Curtis F, et al. Efficacy of supervised maintenance exercise following pulmonary rehabilitation on health care use: a systematic review and meta-analysis. Int J Chron Obstruct Pulmon Dis 2018; 13: 257-273.

9 Ringbaek T, Brondum E, Martinez G, et al. Long-term effects of 1-year maintenance training on physical functioning and health status in patients with COPD: a randomized controlled study. $J$ Cardiopulm Rehabil Prev 2010; 30: 47-52.

10 Soicher JE, Mayo NE, Gauvin L, et al. Trajectories of endurance activity following pulmonary rehabilitation in COPD patients. Eur Respir J 2012; 39: 272-278.

11 Beauchamp MK, Evans R, Janaudis-Ferreira T, et al. Systematic review of supervised exercise programs after pulmonary rehabilitation in individuals with COPD. Chest 2013; 144: 1124-1133.

12 Spencer LM, Alison JA, McKeough ZJ. Maintaining benefits following pulmonary rehabilitation: a randomised controlled trial. Eur Respir J 2010; 35: 571-577.

13 Güell M-R, Cejudo P, Ortega F, et al. Benefits of long-term pulmonary rehabilitation maintenance program in patients with severe chronic obstructive pulmonary disease. Three-year follow-up. Am J Respir Crit Care Med 2017; 195: 622-629.

14 Fan VS, Giardino ND, Blough DK, et al. Costs of pulmonary rehabilitation and predictors of adherence in the National Emphysema Treatment Trial. COPD 2008; 5: 105-116. 
15 Fischer MJ, Scharloo M, Abbink JJ, et al. Drop-out and attendance in pulmonary rehabilitation: the role of clinical and psychosocial variables. Respir Med 2009; 103: 1564-1571.

16 Robinson H, Williams V, Curtis F, et al. Facilitators and barriers to physical activity following pulmonary rehabilitation in COPD: a systematic review of qualitative studies. NPJ Prim Care Respir Med 2018; $28: 19$.

17 Zheng M. Master Cheng's Thirteen Chapters on t'ai-chi ch'üan=[Zhengzi tai ji quan shi san pian]. Brooklyn, NY, Sweet Ch'i Press, 1983.

18 Helm B. Gateways to health: Taijiquan and traditional Chinese medicine. Taijiquan J 2002; 3: 8-12.

19 Taylor-Piliae RE. Tai Chi as an adjunct to cardiac rehabilitation exercise training. J Cardpulm Rehabil 2003; 23: 90-96.

20 Yeh GY, Litrownik D, Wayne PM, et al. BEAM study (Breathing, Education, Awareness, Movement): a randomised controlled feasibility trial of tai chi exercise in patients with COPD. BMJ Open Respir Res 2020; 7 : e000697.

21 Moy ML, Wayne PM, Litrownik D, et al. Long-term exercise after pulmonary rehabilitation (leap): design and rationale of a randomized controlled trial of Tai Chi. Contemp Clin Trials 2015; 45: 458-467.

22 Yeh GY, Wood MJ, Lorell BH, et al. Effects of tai chi mind-body movement therapy on functional status and exercise capacity in patients with chronic heart failure: a randomized controlled trial. Am J Med 2004; 117 : 541-548.

23 Yeh GY, Lorell BH, Stevenson LW, et al. Benefit of Tai Chi as an adjunct to standard care for patients with chronic stable heart failure. J Card Fail 2003; 9: S1.

24 Yeh GY, Wayne PM, Litrownik D, et al. Tai chi mind-body exercise in patients with COPD: study protocol for a randomized controlled trial. Trials 2014; 15: 337.

25 Borg E, Borg G, Larsson K, et al. An index for breathlessness and leg fatigue. Scand J Med Sci Sports 2010; 20: 644-650.

26 ATS Committee on Proficiency Standards for Clinical Pulmonary Function Laboratories. ATS statement: guidelines for the six-minute walk test. Am J Respir Crit Care Med 2002; 166: 111-117.

27 Redelmeier DA, Bayoumi AM, Goldstein RS, et al. Interpreting small differences in functional status: the Six Minute Walk test in chronic lung disease patients. Am J Respir Crit Care Med 1997; 155: 1278-1282.

28 Polkey MI, Spruit MA, Edwards LD, et al. Six-minute-walk test in chronic obstructive pulmonary disease: minimal clinically important difference for death or hospitalization. Am J Respir Crit Care Med 2013; 187: 382-386.

29 Eldridge SM, Chan CL, Campbell MJ, et al. CONSORT 2010 statement: extension to randomised pilot and feasibility trials. BMJ 2016; 355: i5239.

30 Yeh GY, Roberts DH, Wayne PM, et al. Tai chi exercise for patients with chronic obstructive pulmonary disease: a pilot study. Respir Care 2010; 55: 1475-1482.

31 Hedges LV, Olkin I. Statistical Methods for Meta-analysis. Orlando, Academic Press, 1985.

32 Cohen J. Statistical Power Analysis for the Behavioral Sciences. Rev. edn. New York, Academic Press, 1977.

33 Spencer LM, McKeough ZJ. Maintaining the benefits following pulmonary rehabilitation: achievable or not? Respirology 2019; 24: 909-915.

34 Jiménez-Reguera B, Maroto López E, Fitch S, et al. Development and preliminary evaluation of the effects of an mHealth web-based platform (HappyAir) on adherence to a maintenance program after pulmonary rehabilitation in patients with chronic obstructive pulmonary disease: Randomized controlled trial. JMIR MHealth UHealth 2020; 8: e18465.

35 Wu W, Liu X, Wang L, et al. Effects of Tai Chi on exercise capacity and health-related quality of life in patients with chronic obstructive pulmonary disease: a systematic review and meta-analysis. Int J Chron Obstruct Pulmon Dis 2014; 9: 1253-1263.

36 Ding M, Zhang W, Li K, et al. Effectiveness of T'ai Chi and Qigong on chronic obstructive pulmonary disease: a systematic review and meta-analysis. J Altern Complement Med 2014; 20: 79-86.

37 Leung RWM, McKeough ZJ, Peters MJ, et al. Short-form Sun-style t'ai chi as an exercise training modality in people with COPD. Eur Respir J 2013; 41: 1051-1057.

38 Li Y, Feng J, Li Y, et al. A new pulmonary rehabilitation maintenance strategy through home-visiting and phone contact in COPD. Patient Prefer Adherence 2018; 12: 97-104.

39 Wilson AM, Browne $\mathrm{P}$, Olive $\mathrm{S}$, et al. The effects of maintenance schedules following pulmonary rehabilitation in patients with chronic obstructive pulmonary disease: a randomised controlled trial. BMJ Open 2015; 5: e005921. 